\title{
Search for signatures with top, bottom, tau and exotics
}

\author{
Andrea Favareto; Camilla Galloni'and Luigi Longo \\ On behalf of the ATLAS and CMS Collaborations \\ E-mail: andrea.favareto@ge.infn.it, camilla.galloni@cern.ch, \\ luigi.longoele.infn.it
}

\begin{abstract}
The Standard Model of particle physics is a sensational success, especially since the discovery of the $125 \mathrm{GeV}$ Higgs boson. However, there are still several open questions that the Standard Model doesn't address, like the nature of dark matter and dark energy, the matter-antimatter asymmetry, the neutrino oscillations, the inclusion of general relativity and the hierarchy problem. Theories Beyond the Standard Model (BSM), such as Supersymmetry, Little and Composite Higgs, Extra-Dimensions, Extended Gauge models, Technicolor, Left-Right symmetric models, and many other BSM scenarios are trying to answer these questions. In these proceedings we present the most recent results for searches Beyond the Standard Model at the Large Hadron Collider (LHC) by the ATLAS and CMS experiments, focusing on signatures with top, bottom, tau and exotics. The data are found to be consistent with the Standard Model. The non-observation of a signal allows to set limits at the $95 \%$ confidence level on the production cross section times branching ratio and on the masses of the hypothesized new particles for appropriate benchmark models.
\end{abstract}

PP @ LHC 2016 - VII Workshop italiano sulla fisica pp a LHC

16-18 May 2016

Pisa, Italy

\footnotetext{
*Università degli Studi di Genova and INFN, Genova, Italy

$\dagger$ Universitaet Zuerich, Zuerich, Switzerland

¥Università del Salento and INFN, Lecce, Italy
} 


\section{Introduction}

The Standard Model of particle physics has been very successful at describing particles and their interactions. There are however several open questions that the Standard Model doesn't address. The unexplained phenomena and problems like the nature of dark matter and dark energy, the matter-antimatter asymmetry, the neutrino oscillations, the inclusion of general relativity and the hierarchy problem, suggest that the universe we observe requires theoretical developments Beyond the Standard Model (BSM). The Large Hadron Collider (LHC) at CERN has provided the ATLAS [1] and CMS [2] experiments with high energy proton-proton collisions to test the Standard Model and perform searches for physics Beyond the Standard Model. Among the possible extensions of the Standard Model, Supersymmetry (SUSY) [3] provides solutions to some of the open questions. In addition to SUSY, there are many other theoretical approaches grouped under the term of exotic physics (e.g. Little and Composite Higgs, Extra-Dimensions, Extended Gauge models, Technicolor, Left-Right symmetric models, etc.) that address as well some of the open issues mentioned above, and predict a rich phenomenology accessible at the LHC.

The ATLAS and CMS experiments has run extensive physics searches to cover as many scenarios as possible. These proceedings review a selection of results among the many searches for supersymmetry and exotic signatures based on the LHC proton-proton collision data collected in Run $1^{1}$ at centre of mass energy of 7 and $8 \mathrm{TeV}$, and Run $2^{2}$ at centre of mass energy of $13 \mathrm{TeV}$.

\section{Supersymmetry}

Supersymmetry is one of the most studied framework to extend the Standard Model since it provides a natural solution to the hierarchy problem. In its minimal formulation, Minimal Supersymmetric Standard Model (MSSM), it predicts a new bosonic (fermionic) partner for each fermionic (bosonic) Standard Model particle with identical quantum number except for a difference of half unit of spin $(S)$. An additional Higgs doublet is also introduced together with its supersymmetric partner. In order to preserve the baryon number (B) and lepton number(L), it is assumed that the multiplicative quantum number $R$-parity, defined as $R=(-1)^{3(B-L)+2 S}$, is conserved. In this framework SUSY particles are produced in pairs and the lightest supersymmetric particle (LSP) is stable and a candidate for dark matter.

The SUSY partners of the Higgs and electroweak gauge bosons mix to form the mass eigenstates $^{3}$ known as charginos $\left(\tilde{\chi}_{i=1,2}^{ \pm}\right)$and neutralinos $\left(\tilde{\chi}_{i=1,2,3,4}^{0}\right)$; in a large variety of models, the LSP is the lightest neutralino $\tilde{\chi}_{1}^{0}$ and events with large missing momentum, $E_{\mathrm{T}}^{\text {miss }}$, are searched as proof of the supersymmetry. Even the scalar partners of the right-handed and left-handed top (bottom), $t(b)$ quark, $\tilde{t}_{R}$ and $\tilde{t}_{L}\left(\tilde{b}_{R}\right.$ and $\tilde{b}_{L}$ ), can mix to form two mass eigenstates, $\tilde{t}_{1}$ and $\tilde{t}_{2}\left(\tilde{b}_{1}\right.$ and $\tilde{b}_{2}$ ). In many scenarios $\tilde{t}_{1}$ is assumed to be the lightest supersymmetric quark (squark) and as

\footnotetext{
${ }^{1}$ Run 1 corresponds to LHC proton-proton data from years 2011 and 2012 at the centre of mass energies of $7 \mathrm{TeV}$ and $8 \mathrm{TeV}$. The collision datasets correspond to approximately $5 \mathrm{fb}^{-1}$ at centre of mass energy of $7 \mathrm{TeV}$ and $20 \mathrm{fb}^{-1}$ at $8 \mathrm{TeV}$.

${ }^{2}$ Run 2 corresponds to LHC proton-proton data from 2015, hence after the machine technical stop of 2013-2014, at the centre of mass energy of $13 \mathrm{TeV}$. The collision datasets correspond to approximately $3 \mathrm{fb}^{-1}$ so far.

${ }^{3}$ All the mass eigenstates are ordered by increasing mass.
} 


\begin{tabular}{lrr}
\hline \hline Signal Model & SUSY masses spectrum & Experiment \\
\hline \hline$\tilde{g} \rightarrow t \bar{t} \tilde{\chi}_{1}^{0}$ & $m(\tilde{g})<<m(\tilde{q})$ & ATLAS [4, 5] \& CMS [6] \\
$\tilde{g} \rightarrow b \bar{b} \tilde{\chi}_{1}^{0}$ & $m(\tilde{g})<<m(\tilde{q})$ & ATLAS [5] \& CMS [6] \\
$\tilde{g} \rightarrow \tilde{t}_{1} \bar{t} \rightarrow \bar{t} \tilde{\chi}_{1}^{0}$ soft term & $\Delta m\left(\tilde{t}_{1}, \tilde{\chi}_{1}^{0}\right)=5 \mathrm{GeV}$ & ATLAS [7] \\
\hline$\tilde{t}_{1} \rightarrow t \tilde{\chi}_{1}^{0}$ & $m\left(\tilde{t}_{1}\right)>m\left(\tilde{\chi}_{0}^{ \pm}\right)$ & ATLAS [7] \& CMS [8, 9] \\
$\tilde{t}_{1} \rightarrow b \tilde{\chi}_{1}^{ \pm} \rightarrow b W \tilde{\chi}_{1}^{0}$ & $m\left(\tilde{t}_{1}\right)=m\left(\tilde{\chi}_{1}^{ \pm}\right)+10 \mathrm{GeV}$ & ATLAS [10] \\
$\tilde{t}_{1} \rightarrow b \tilde{\chi}_{1}^{ \pm} \rightarrow b W \tilde{\chi}_{1}^{0}$ & $m\left(\tilde{\chi}_{1}^{ \pm}\right)=2 \times m\left(\tilde{\chi}_{0}^{ \pm}\right)$ & ATLAS [10] \\
\hline$\tilde{b}_{1} \rightarrow b \tilde{\chi}_{1}^{0}$ & $m\left(\tilde{b}_{1}\right)>m\left(\tilde{\chi}_{0}^{ \pm}\right)$ & ATLAS [11] \& CMS [12] \\
$\tilde{b}_{1} \rightarrow t \tilde{\chi}_{1}^{ \pm} \rightarrow t W \tilde{\chi}_{1}^{0}$ & $m\left(\tilde{\chi}_{1}^{ \pm}\right)=m\left(\tilde{\chi}_{1}^{0}\right)+100 \mathrm{GeV}$ & ATLAS [4] \\
\hline
\end{tabular}

Table 1: SUSY models analyzed by ATLAS and CMS with a final state characterized by a top quark or bottom quark signature. For each of them it has been specified the SUSY masses spectrum assumed and which experiment has developed analys(es). A 100\% Branching Ratio (BR) is assumed for all the decays.

a consequence bottom squark may also be light, being bound to top squaks by $S U(2)$ invariance leading to a potentially large production cross-sections at the Large Hadron Collider (LHC).

Thanks to the increased Run 2 LHC centre of mass energy (13 TeV), the sensitivity to a possible discovery is higher than the one reached in Run 1. For this reason, both ATLAS and CMS experiments were able to explore the possibility of a supersymmetry discovery already with the data collected in 2015 , consisting in an integrate luminosity of $3.2 \mathrm{fb}^{-1}$ and $2.3 \mathrm{fb}^{-1}$ respectively for the two experiments. In particular, focusing the attention on SUSY processes characterized by signatures with top quarks $(t)$ and bottom quarks $(b)$ in the final state, the studied signal models essentially involve the pair production of gluinos $(\tilde{g})$, top squaks $\left(\tilde{t}_{1}\right)$ and bottom squarks $\left(\tilde{b}_{1}\right)$ as shown in Tab. 1.

To better discriminate the supersymmetric processes from the Standard Model ones, in addition to the missing transverse momentum, other kinematic variables based on or correlated to $E_{\mathrm{T}}^{\text {miss }}$ were used. For example in the CMS multi-jets analysis [6] that looks at $\tilde{g} \rightarrow t \bar{t} \tilde{\chi}_{1}^{0}$ and $\tilde{g} \rightarrow b \bar{b} \tilde{\chi}_{1}^{0}$ decay processes, due to the all hadronic-final state request, the target SUSY events are expected to exhibit large values of $H_{T}$ and $H_{T}^{\text {miss }}$; while $H_{T}$ is the scalar sum of the transverse momenta of the jets, $H_{T}^{\text {miss }}$ is the magnitude of the vector sum of the jet $p_{T}$ and can be considered an estimate of the missing transverse momentum when the $E_{\mathrm{T}}^{\text {miss }}$ soft term ${ }^{4}$ is neglected; in this way a better separation between signal and background should be obtained since only the neutralinos should contribute to $H_{T}^{\text {miss }}$. Other useful variables used for other searches, like the $\tilde{t}_{1} \rightarrow b \tilde{\chi}_{1}^{ \pm}$[10] or $\tilde{b}_{1} \rightarrow b \tilde{\chi}_{1}^{0}$ [11] ATLAS analyses, were the leptonic stransverse mass, $m_{T 2}$, and the contransverse mass, $m_{C T}$, defined as

$$
\begin{aligned}
m_{T 2}\left(\text { lept }_{1}, \text { lept }_{2}\right) & =\min _{\vec{q}_{t}+\vec{r}_{t}=\vec{P}_{t}^{\text {miss }}}\left\{\max \left[m_{T}\left(\vec{P}_{t}^{\text {lept }}, \vec{q}_{t}\right), m_{T}\left(\vec{P}_{t}^{\text {lept }}, \vec{r}_{t}\right)\right]\right\} \\
m_{C T}\left(\text { jet }_{1}, \text { jet }_{2}\right) & =\sqrt{\left[E_{T}^{\text {jet }}+E_{T}^{\text {jet }}\right]^{2}-\left[\vec{P}_{T}^{\text {jet }}-\vec{P}_{T}^{\text {jet }}\right]^{2}}
\end{aligned}
$$

where $P_{T}^{\text {lept }}$ and $P_{T}^{\text {jet }}$ are the transverse momentum of the $i^{\text {th }}$ lepton (lept) and the $j^{\text {th }}$ jet respec-

\footnotetext{
${ }^{4}$ the $E_{\mathrm{T}}^{\text {miss }}$ soft term takes into account the calorimeter deposit not associated with any objects.
} 
tively ${ }^{5}$ while $m_{T}$ indicates the transverse mass ${ }^{6} . m_{T 2}$ could be considered to be a variable formed from dividing $\vec{P}_{t}^{\text {miss }}$ into two parts $\left(\vec{q}_{t}, \vec{r}_{t}\right)$ in all possible combinations that satisfy the kinematics of the event and calculating the transverse mass for each decay branch; the resulting value is the best lower limit on the mass of a pair-produced SUSY particle that could have decayed to the observed final state with the given $\vec{q}_{t}, \vec{r}_{t}$ and $\vec{P}_{t}^{\text {miss }}$; for $t \bar{t}$ and $W W$ decays, $m_{T 2}$ is bounded from above by the mass of the $\mathrm{W}$ boson while $\tilde{t}_{1}$ processes are not limited. $m_{C T}$ instead has a bound at $135 \mathrm{GeV}$ for $t \bar{t}$ events whereas for production of bottom squark pairs the limit is given by $\left(m_{\tilde{b}_{1}}^{2}-m_{\tilde{\chi}_{0}^{1}}^{2}\right) / m_{\tilde{b}_{1}}$; in this way for a signal model with a bottom squark mass of $800 \mathrm{GeV}$ and a neutralino massless the upper-bound is pushed up to $800 \mathrm{GeV}$.

For data interpretation the two experiments use a different approach. CMS prefers to define several orthogonal Signal Regions and to combine them in case no excess is observed. For instance this is the case of the CMS multi-jets analysis where 72 signal regions were defined considering different ranges of jet multiplicity $\left(N_{\text {jet }}\right)$, asking for a different number of b-jet $\left(N_{b-j e t}\right)$ and then defining 6 regions in the $H_{T}-H_{T}^{\text {miss }}$ plane as shown in Fig. 1. In contrast with this choice, in
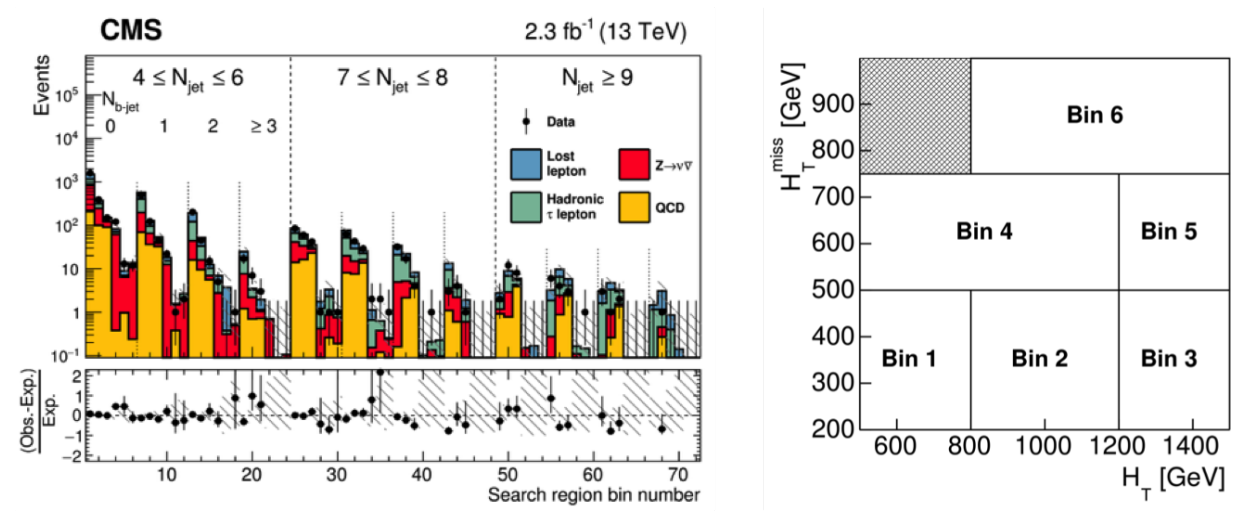

Figure 1: In the left plot the observed numbers of events and the corresponding Standard Model background predictions in the 72 signal regions defined by CMS for the search of $\tilde{g} \rightarrow t \bar{t} \tilde{\chi}_{1}^{0}$ and $\tilde{g} \rightarrow b \bar{b} \tilde{\chi}_{1}^{0}$. On the right the definition of the 6 regions in the $H_{T}-H_{T}^{\text {miss }}$ plane [6].

the ATLAS analyses it is possible to have the definition of different non-orthogonal SRs and only the one with the best sensitivity is considered for the final results. For example for the $\tilde{t}_{1} \rightarrow b \tilde{\chi}_{1}^{ \pm}$ analysis to look to bottom squark models, where a large mass difference between $\tilde{b}_{1}$ and $\tilde{\chi}_{1}^{0}$ is present, three signal regions where defined just increasing the cut on the $m_{C T}$ variables leading to a not statistically independent definition of the SRs; a choice between them is therefore obliged to avoid bias in the analysis.

With exception of the ATLAS $\tilde{t}_{1} \rightarrow t \tilde{\chi}_{1}^{0}$ analysis, where a $2.3 \sigma$ excess has been observed ${ }^{7}$ in one of the signal regions, for all the analysis shown in Tab. 1 no significant excess has been observed and, as a consequence, limits on the SUSY particles masses have been set at 95\% CL. In

\footnotetext{
${ }^{5}$ Leptons and jets are sorted with respect to their transverse momenta, from the most energetic to the lowest one.

${ }^{6}$ The transverse mass $m_{T}$ is defined as $m_{T}^{2}\left(\vec{P}_{T}^{\alpha}, \vec{P}_{T}^{m i s s}, m_{\alpha}, m_{\chi}\right)=m_{\alpha}^{2}+m_{\chi}^{2}+2\left(E_{T}^{\alpha} E_{\mathrm{T}}^{\text {miss }}-\vec{P}_{T}^{\alpha} \cdot \vec{P}_{T}^{m i s s}\right)$ where $E_{T}^{\alpha}$ is equal to $\sqrt{\left|\vec{P}_{T}^{\alpha}\right|^{2}+m_{\alpha}^{2}}, E_{T}^{\text {miss }}$ is defined as $\sqrt{\left|\vec{P}_{T}^{m i s s}\right|^{2}+m_{\chi}^{2}}, m_{\alpha}$ and $\vec{p}_{T}^{\alpha}$ are the mass and transverse momentum of some visible particle. The parameter $m_{\chi}$ is the mass of the invisible particle, which is usually assumed to be zero.

${ }^{7} 12$ events were observed, against the expected $5.50 \pm 0.72$.
} 
Fig. 2 the exclusion limits for $\tilde{g} \rightarrow t \bar{t} \tilde{\chi}_{1}^{0}, \tilde{g} \rightarrow b \bar{b} \tilde{\chi}_{1}^{0}, \tilde{t}_{1} \rightarrow b \tilde{\chi}_{1}^{ \pm}$and $\tilde{b}_{1} \rightarrow b \tilde{\chi}_{1}^{0}$ processes are shown together with the Run 1 results when available.

All the results provided by the two collaboration are valid only under the assumption of simplified models with a branching ratio equal to $100 \%$. In fact, if additional decays processes are present in the theory, worse exclusion limits can be obtained due to the fact that less signal events are expected in the SR because of the lower branching ratio.
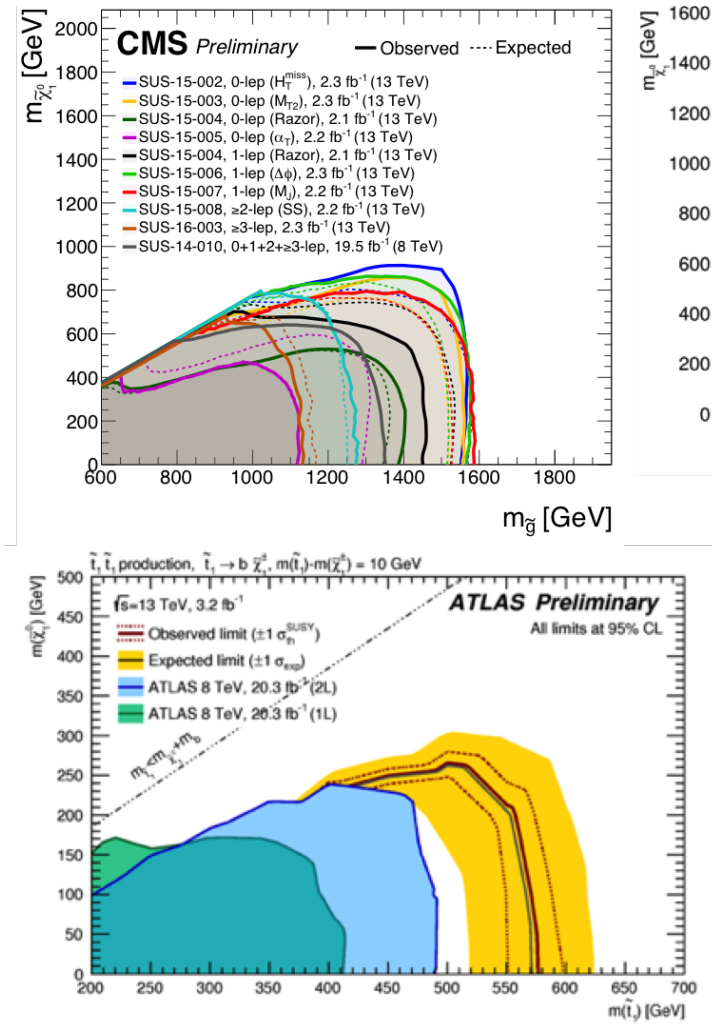
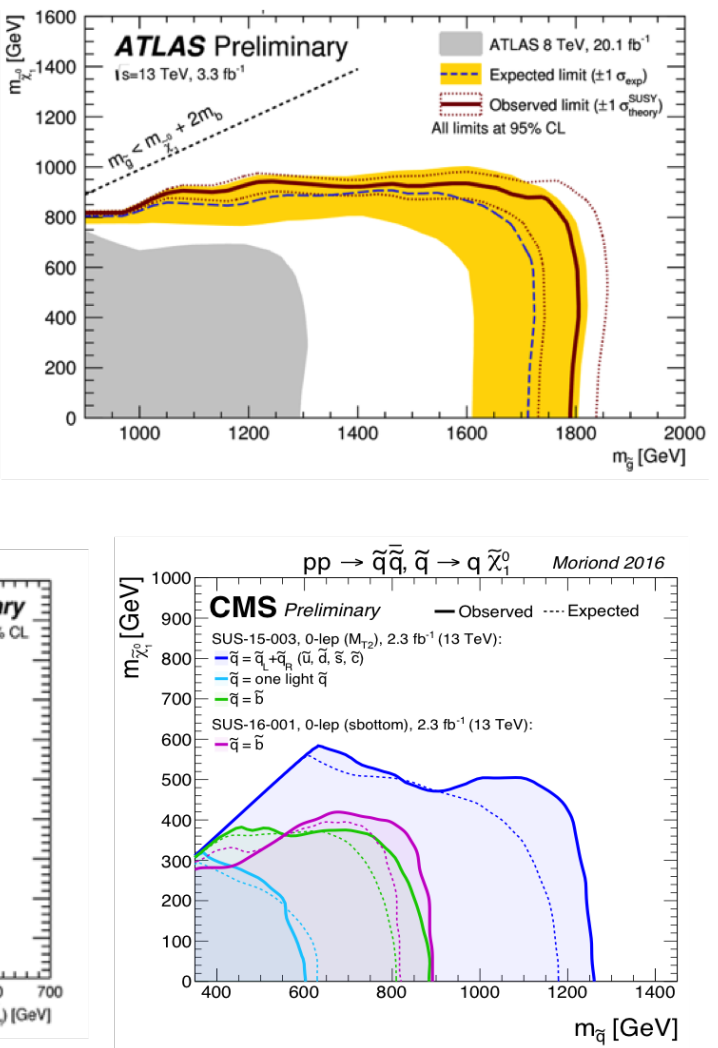

Figure 2: In the top part of the figure the summary of exclusion limits relative to $\tilde{g} \rightarrow t \bar{t} \tilde{\chi}_{1}^{0}$ signal model obtained by the CMS experiment (left) [14] is shown together with the exclusion plot relatives to the $\tilde{g} \rightarrow$ $b \bar{b} \tilde{\chi}_{1}^{0}$ process obtained by the ATLAS experiment (right) [5]. In the bottom part the ATLAS limits for $\tilde{t}_{1} \rightarrow b \tilde{\chi}_{1}^{ \pm}$[10], under the assumption that $m\left(\tilde{t}_{1}\right)=m\left(\tilde{\chi}_{1}^{ \pm}\right)+10 \mathrm{GeV}$, are reported together with the CMS ones for the $\tilde{b}_{1} \rightarrow b \tilde{\chi}_{1}^{0}$ decay [14] .

\section{Vector like quarks and resonances}

Vector like quarks (VLQ) are non chiral fermions predicted by a large variety of theories BSM, like Little Higgs [15] and Composite Higgs [16] . They are defined as colour-triplet spin-1/2 fermions whose left- and right-handed chiral components have the same transformation properties under the weak-isospin SU(2) gauge group [17]. These particles can be SU(2) singlets or doublets and have the same charge as the top or bottom quarks $(T, B)$ or exotic charges $5 / 3$ or $-4 / 3\left(X_{5 / 3}\right.$, $\left.Y_{-4 / 3}\right)$. Vector-like quarks are expected to couple preferentially to third-generation quarks and they can have both charged and flavor-changing neutral current decays. 
Final states with heavy flavor quarks and third generation leptons are interesting also for other numerous extensions to the Standard Model, that predict gauge interactions with enhanced couplings to third generation quarks and leptons. Massive new particles could manifest as resonances in the production of Standard Model particles pairs. Examples of such resonances include models with massive color-singlet $Z$-like or $W$-like bosons in extended gauge theories [18], colorons [19], axigluons [20] and models with extra dimensions, such as Kaluza-Klein (KK) excitations of gluons, electroweak gauge bosons or gravitons in various extensions of the Randall-Sundrum (RS) model [21]. These models predict resonances in the TeV mass range. Resonant Standard Model object production could be visible in the reconstructed invariant mass spectrum of the final state system. In this TeV range, both ATLAS and CMS experiments, thanks to the increase in the LHC Run 2 centre of mass energy, have the same sensitivity to new processes with the dataset collected in 2015 as the one collected in Run 1. Thus, many analysis performed in Run 1 were optimized and performed again with the 2015 data.

The decay products of such high masses resonances can be boosted, i.e. have large momentum compared to their masses, so that their final decay products can be very collimated. This means for hadronic quarks and bosons decays that a single hadronic jet with a large radius encompasses all its decay products. Techniques that exploit the presence of substructure within this jet are employed to reject background from standard QCD jets and reduce the dependence on multiple interactions within the same bunch crossing (pile-up). Despite differences in the algorithms, ATLAS and CMS employ similar tools to identify (tag) jets compatible with top-quark, $W, Z$ or Higgs bosons decays in boosted topologies [22, 23, 24, 25, 26, 27].

\subsection{Vector like quarks}

For pair produced vector like quark of charge $2 / 3(T)$, with $T \rightarrow W b, Z t, H t$, both experiments have update the Run 1 results with the data collected in 2015. The ALTAS collaboration performed a search [28] for VLQ $T$-pair production with significant branching ratio to a Higgs boson and a top quark, $T T \rightarrow H t+X$ in the lepton plus jets final state, characterised by an isolated electron or muon with high transverse momentum, large missing transverse momentum and multiple jets. The search exploits the high multiplicity of $b$-jets, the high scalar sum of transverse momenta of all final state objects, and the presence of boosted, hadronically-decaying resonances reconstructed as large-radius jets. The CMS search [29] selects events in the lepton plus jets final state with large number of jets and at least a large cone jet, which is $W$-tagged, using as main discriminating variable the minimal invariant mass between all the combinations of the lepton and a $b$-tagged jets. For both experiments, no significant excess of events above the Standard Model expectation is observed, and $95 \% \mathrm{CL}$ lower limits are derived on the mass of the vector-like $T$ quark under several branching ratio hypotheses assuming contributions only from $T \rightarrow W b, Z t, H t$. ATLAS and CMS observe 95\% confidence level (CL) lower limits on the $T$ quark mass range between $700 \mathrm{GeV}$ and $900 \mathrm{GeV}$ for all possible values of the branching ratios into the three decay modes, already improving the Run 1 limits of about 50-100 GeV

$T$ quarks can be also singly produced assuming $W b$ and $Z t$ coupling in production. A search [30] was performed by the CMS collaboration in lepton plus jet final state. Since the decay of a vectorlike $T$ quark into a Higgs boson and a top quark is searched for, events are selected requiring at least a Higgs-tagged jet and the invariant mass spectrum of the $T$ system is reconstructed and inves- 
tigated for "bumps" compatible with new resonances. No significant excess over Standard Model backgrounds is observed. For a mass of $1000 \mathrm{GeV}$, production cross sections times branching fractions above $0.9 \mathrm{pb}$ and $0.6 \mathrm{pb}$ are excluded at $95 \%$ confidence level, assuming left and right handed coupling of the $T$ quark to Standard Model particles, respectively.

Other heavy top quark partners of charge 5/3 are looked for with data collected by CMS [31]. The search exploits events with two different signatures: same-sign dileptons as well as a semileptonic final state. No significant excess is observed in the data and observed 95\% CL exclusion limits on the mass of a right handed and left handed $X$ quark are set to be $960 \mathrm{GeV}$ and $940 \mathrm{GeV}$, respectively.

\subsection{Resonances}

Both ATLAS and CMS search for heavy $Z$ boson like resonances $\left(Z^{\prime}\right.$ s) decaying to top-antitop quark pairs in the 2015 data, looking for one top quark decaying hadronically while the other decays leptonically $[32,33]$. Both selections are optimized for heavy resonance masses, where the top quarks have large Lorentz-boosts. This results in non-isolated leptons from the $W \rightarrow \ell v$ decay and two or more quarks reconstructed in a single jet from the $t \rightarrow b W \rightarrow b q q^{\prime}$ decay. These jets are identified using top-tagging algorithms. The $Z^{\prime}$ boson reconstruction differs between the two experiments: CMS assigns the lepton and the jet to the leptonically decaying top and the jets to the hadronically decaying one minimizing a $\chi^{2}$ function that keeps into account the top mass resolution, evaluated in simulations; ATLAS instead chooses to assign to the leptonic top the closest jet to the lepton. No evidence for resonant $t \bar{t}$ production is found and upper limits on the production cross section of heavy resonances for various width and physics models are set. In the narrow width scenarios, limits are set between $100 \mathrm{pb}$ and $70 \mathrm{fb}$ for masses between $500 \mathrm{GeV}$ and $4 \mathrm{TeV}$ (CMS) or $5 \mathrm{TeV}$ (ATLAS), showing how the two experiments reach very similar sensitivities.

Resonances compatible with a heavy gauge $W^{\prime}$ boson decaying to top and bottom quarks and $\tau \nu$ leptons have been performed by CMS with the 2015 dataset. In the search [34] for $W^{\prime} \rightarrow t b$, a final state signature with a lepton $(e, \mu)$, jets, and missing transverse energy is analyzed. Events are further divided in categories depending on the number of $b$-tagged events, being events with no $b$-tagged jets enriched in backgrounds. A bump in the invariant mass spectrum of the $W^{\prime}(t b)$ is searched. No evidence of a $W^{\prime}$ is found and 95\% CL upper limits on its production cross-section times branching fraction are set. For $W^{\prime}$ bosons with purely right handed couplings, the observed 95\% CL lower limit is $2.4 \mathrm{TeV}$, being currently the most stringent limits in this channel.

In the search [35] for $W^{\prime} \rightarrow \tau \nu$, a signal would appear as an excess of events with high transverse mass, where the Standard Model background is low. Events are selected requiring an hadronic tau and missing energy compatible with the neutrino presence. To suppress background from QCD jets faking tau leptons, isolation requirements are applied in cones around the hadronic tau candidates. At high masses the limits obtained from the 2015 data are already more stringent, but for masses below $1 \mathrm{TeV}$ the Run 1 limits still profits from the larger statistics of 2012 data. Combining the two analyses, sequential Standard Model $W^{\prime}$ can be excluded in the mass range from $300 \mathrm{GeV}$ to $3.3 \mathrm{TeV}$ at $95 \% \mathrm{CL}$. 


\section{Long-Lived Particles searches}

Massive, long-lived particles (LLPs) are predicted by a wide range of physics models that extend the Standard Model. LLPs arise in proposed solutions to the gauge hierarchy problem, including supersymmetric models that either violate or conserve $R$-parity. The lifetime of these particles depends on the mass difference between the particle and the lightest stable SUSY particle, and on the size of any $R$-parity-violating coupling. Because of their large mass, LLPs are expected to be slow ( $\beta$ significantly below 1 ) and, if charged, to have a specific ionization higher than any Standard Model particles of unit charge at high momenta.

Long-lived particles, with lifetimes from around $1 \mathrm{~ns}$ to several tens of ns, can either travel distances comparable to the size of the ATLAS and CMS detectors and thus appear to be stable, or can decay far from the primary interaction point. Wide-ranging searches for LLPs are performed by both experiments exploiting different techniques, with some nontrivial overlaps in lifetime coverage.

\subsection{Stable and meta-stable Massive particles}

As mentioned before, heavy LLPs are predicted by several extensions of the Standard Model. Some Supersymmetry models [36] predict the existence of meta-stable sleptons, in particular in Gauge-Mediated SUSY Breaking (GMSB) with the $\tau$ slepton $(\tilde{\tau})$ as the LLP, and of colored metastable squarks $(\tilde{q})$ and gluinos $(\tilde{g})$ in split SUSY. Squarks and gluinos can hadronize with either a light Standard Model quark system or a gluon, forming a $R$-hadron. $R$-hadrons may be singly charged, doubly charged or neutral, they can either change their electric charge by nuclear scattering processes with the detector material, or decay in the detector given their finite lifetime. Heavy LLPs should be produced at the LHC as massive particles. They are expected to move slowly $(\beta<1)$, thereby having a measurable time-of-flight, and to release an anomalous amount of energy while passing through the detector. They may also interact like heavy muons.

According to these signatures, ATLAS and CMS performed several searches. ATLAS recently published, with the 2015 LHC data at $\sqrt{s}=13 \mathrm{TeV}$ corresponding to an integrated luminosity of $3.2 \mathrm{fb}^{-1}$, an Inner Detector (ID)-only search, potentially sensitive to meta-stable gluino $R$-hadrons not reaching farther sub-detectors, or to stable gluino $R$-hadrons [37]. The Pixel subsystem of the ATLAS detector is used to measure the ionization energy loss of reconstructed charged particles and to search for such highly ionizing particles. No significant deviation from Standard Model background expectations is observed. Gluino R-hadrons with lifetimes above $0.4 \mathrm{~ns}$ and decaying to $q \bar{q}$ plus a $100 \mathrm{GeV}$ neutralino are excluded at the $95 \%$ confidence level, with lower mass limit ranging between $740 \mathrm{GeV}$ and $1590 \mathrm{GeV}$. In the case of stable $R$-hadrons the lower mass limit at the $95 \%$ confidence level is $1570 \mathrm{GeV}$. ATLAS and CMS experiments are also performing fulldetector searches, mainly intended for stable R-hadrons. In particular CMS published a search for heavy stable charged particles produced in pp collisions at $\sqrt{s}=13 \mathrm{TeV}$ corresponding to an integrated luminosity of $2.4 \mathrm{fb}^{-1}$ [38]. Candidates are asked to have signatures of anomalously high energy deposition in the silicon tracker and long time-of-flight to the muon detectors. The data are consistent with the expected background and limits on the cross section for production of long-lived gluinos, stops and staus are set. Corresponding lower mass limits, ranging up to $1590 \mathrm{GeV}$ for gluinos, are the most stringent to date. 


\subsection{Disappearing tracks}

In Anomaly-Mediated Supersymmetry Breaking (AMSB) models, the lightest chargino is nearly mass degenerate with the lightest neutralino. One prominent feature of these scenarios is that the chargino has a considerable lifetime and predominantly decays into a neutralino plus a low-momentum charged pion. The low-momentum charged pion track is rarely reconstructed due to its large displacement and a small number of interactions in the tracking system, therefore, a decaying chargino is typically recognized as a "disappearing track" that has few associated hits in the outer tracking volume. Ad-hoc reconstruction algorithms for short tracks are needed, because finding the disappearing-track signature suffers from charged hadrons interacting in the detector material, charged leptons losing much of their momenta due to scattering with material or large bremsstrahlung, and short-length tracks originating from a combination of wrong space-points and resulting in anomalously high values $p_{T}$.

Two searches for direct chargino production based on a disappearing-track signature, using $20.3 \mathrm{fb}^{-1}$ of proton-proton collisions at $\sqrt{s}=8 \mathrm{TeV}$ collected at the LHC, have been performed by ATLAS [39] and CMS [40]. No significant excess above the background expectation is observed for candidate tracks with large transverse momentum, and constraints on chargino properties are obtained. In the AMSB scenarios, ATLAS and CMS excluded at $95 \%$ confidence level a chargino mass below $270 \mathrm{GeV}$ and $260 \mathrm{GeV}$ respectively.

\subsection{Displaced vertex}

Many extensions to the Standard Model include the production of particles that are neutral, weakly-coupled and long-lived that can decay to final-states containing several hadronic jets. In particular, three models are studied with the ATLAS experiment:

- a Standard Model sector scalar boson $\Phi$ mixes with $\Phi_{h s}$, a hidden sector scalar boson which can decay to $v$-quarks $\left(q_{v}\right)$. The hidden sector consists of a confining gauge group that makes $v$-hadrons out of its $v$-quarks, in analogy with QCD. The $v$-quarks then hadronize to $v$-particles that can decay back to Standard Model particles. The lightest hidden sector particles $\pi_{v}$ are pair-produced and each decays to a pair of Standard Model fermions. The lifetime of the $\pi_{v}$ is unconstrained and could be quite long. If a $\pi_{v}$ decays in the Inner Detector (ID) or in the Muon Spectrometer (MS), it can be reconstructed as a non-standard displaced vertex (DV). A $\pi_{v}$ that instead decays deep inside the calorimeter is reconstructed as a jet with an unusual energy signature that most traditional searches reject as having poor data quality.

- a massive communicator, $Z^{\prime}$, produced by quark-antiquark annihilation decays into the hidden sector via $Z^{\prime} \rightarrow q_{v} \bar{q}_{v}$. The $v$-quarks hadronize into showers of $\pi_{v}$ particles. The $\pi_{v}$ lifetime is a free parameter.

- Stealth SUSY: a class of $R$-parity-conserving SUSY models that do not have large missing transverse momentum signatures. This scenario results in one prompt gluon and two displaced gluon jets per gluino decay resulting in two DVs.

ATLAS performed two searches for the decay of neutral, weakly interacting, long-lived particles using $20.3 \mathrm{fb}^{-1}$ of 2012 data collected at $\sqrt{s}=8 \mathrm{TeV}$. The first analysis is sensitive to long-lived 
particles that decay to Standard Model particles producing jets at the outer edge of the ATLAS electromagnetic calorimeter or inside the hadronic calorimeter [41]. Events that contain jets with large energy deposit in the hadronic calorimeter and isolated with respect to ID-tracks are selected. In addition a dedicated trigger based on large ratio between the energy deposit in the hadronic calorimeter and the one in the electromagnetic calorimeter $\left(E_{H C a l} / E_{E M}\right)$ is used. The second search employs "non-standard" techniques for reconstructing decay vertices of long-lived particles decaying to jets in the inner detector and muon spectrometer [42]. Secondary vertices in the ID are required to have high track multiplicity and high mass. In addition the secondary vertices must not come from interactions inside the detector material. The MS track segments are required to be isolated with respect to the ID ones and to have high number of hits.

Similar analyses, based on the presence of displaced vertices, has been published also by CMS, searching for long-lived particles decaying to final states that include di-leptons [43] or decaying to di-jets [44]. The data sample used correspond to $20.5 \mathrm{fb}^{-1}$ and $18.6 \mathrm{fb}^{-1}$ respectively of 2012 data collected at $\sqrt{s}=8 \mathrm{TeV}$.

No significant excess of events over the expected background are found, and limits as a function of proper lifetime are reported for the decay of the Higgs boson and other scalar bosons to long-lived particles and for Z' and Stealth SUSY benchmark models.

\subsection{Lepton-Jets}

Several possible extensions of the Standard Model predict the existence of a hidden sector that is weakly coupled to the visible one. Depending on the structure of the hidden sector and its coupling to the Standard Model, some unstable hidden states may be produced at colliders and decay back to Standard Model particles with sizeable branching fractions. In the scenario where the hidden sector and the Standard Model couple via the vector portal, a light hidden photon (dark photon, $\gamma_{d}$ ) mixes kinetically with the Standard Model photon. If the hidden photon is the lightest state in the hidden sector, it decays back to Standard Model particles. From $\gamma_{d}$ decays, collimated jet-like structures containing pairs of electrons and/or muons and/or charged pions (Lepton-Jets, LJs), and which are produced far from the primary vertex of the event, may arise.

A search for LJs in a sample of $20.3 \mathrm{fb}^{-1}$ of proton-proton collisions at $\sqrt{s}=8 \mathrm{TeV}$ collected during 2012, has been performed by the ATLAS experiment and described in detail in [45]. Three topologies are defined. The first topology select LJs with all $\gamma_{d}$ decaying to muons, with $\gamma_{d}$ decays beyond the Pixel Detector up to the first trigger plane of the MS. The second topology select LJs with one $\gamma_{d}$ decaying to a muon pair and one $\gamma_{d}$ decaying to an electron/pion pair. The range of decay distances targeted by this topology extends from the last ID pixel layer up to the end of the Hadronic Calorimeter, for $\gamma_{d}$ decaying into an electron/pion pair, and from the last ID pixel layer up to the first trigger plane of the MS, for the $\gamma_{d}$ decays to muons. The last topology select all $\gamma_{d}$ decaying to electron/pion pairs in the Hadronic Calorimeter. The requirement of low electromagnetic fraction is needed to reduce the Standard Model multi-jet background. Since observed events are consistent with background expectations, upper limits are derived as a function of the LLP $c \tau$.

A similar analysis has been performed by CMS that published a search for pair production of 
third-generation scalar leptoquarks ${ }^{8}$ in a sample of $19.7 \mathrm{fb}^{-1}$ of proton-proton collisions at $\sqrt{s}=$ $8 \mathrm{TeV}$ [46]. The number of observed events is found to be in agreement with the expected standard model background. Third-generation scalar leptoquarks with masses below $740 \mathrm{GeV}$ are excluded at $95 \%$ confidence level, assuming a $100 \%$ branching fraction for the leptoquark decay to a $\tau$ lepton and a bottom quark.

\section{Conclusions}

ATLAS and CMS collaborations are conducting a wide range of searches for BSM processes, exploiting different signatures and using various detection techniques. These proceedings give a summary of the recent results of these searches, focusing on signatures with top, bottom, tau and exotics. The data collected so far are found to be consistent with Standard Model expectations and the non-observation of signals allows to set limits on production cross section times branching ratios and on the masses of the predicted new particles for many BSM benchmark models.

\section{References}

[1] ATLAS Collaboration, The ATLAS Experiment at the CERN Large Hadron Collider, JINST 3 (2008) S08003.

[2] CMS Collaboration, The CMS experiment at the CERN LHC, JINST 3 (2008) S08004.

[3] S. P. Martin, A Supersymmetry Primer, arXiv:hep-ph/9709356 [hep-ph].

[4] ATLAS Collaboration, Search for supersymmetry at $\sqrt{s}=13 \mathrm{TeV}$ in final states with jets and two same-sign leptons or three leptons with the ATLAS detector, arXiv:1602.09058.

[5] ATLAS Collaboration, Search for gluino-mediated stop and sbottom pair production in events with $b$-jets and large missing transverse momentum in $\sqrt{s}=13 \mathrm{TeV}$ pp collisions with the ATLAS detector, ATLAS-CONF-2015-067.

[6] CMS Collaboration, Search for supersymmetry in the multijet and missing transverse momentum final state in pp collisions at $13 \mathrm{TeV}$, arXiv:1602.06581.

[7] ATLAS Collaboration, Search for top squarks in final states with one isolated lepton, jets, and missing transverse momentum in $\sqrt{s}=13 \mathrm{TeV}$ pp collisions of ATLAS data, ATLAS-CONF-2016-007.

[8] CMS Collaboration, Search for new physics with the MT2 variable in all-jets final states produced in pp collisions at $\sqrt{s}=13 \mathrm{TeV}$, arXiv: 1603.04053 .

[9] CMS Collaboration, Search for direct top squark pair production in the single lepton final state at $\sqrt{s}=13 \mathrm{TeV}$, CMS-PAS-SUS-16-002.

[10] ATLAS Collaboration, Search for direct top squark pair production in final states with two leptons in $\sqrt{s}=13 \mathrm{TeV}$ pp collisions using 3.2 $\mathrm{fb}^{-1}$ of ATLAS data, ATLAS-CONF-2016-009.

[11] ATLAS Collaboration, Search for Bottom Squark Pair Production with the ATLAS Detector in proton-proton Collisions at $\sqrt{s}=13 \mathrm{TeV}$, ATLAS-CONF-2015-066.

\footnotetext{
${ }^{8}$ Many extensions of the standard model predict new scalar or vector bosons, called leptoquarks (LQ), which carry non-zero lepton and baryon numbers, as well as color and fractional electric charge
} 
[12] CMS Collaboration, Search for direct production of bottom and light top squark pairs in proton-proton collisions at $\sqrt{s}=13 \mathrm{TeV}$, CMS-PAS-SUS-16-001.

[13] ATLAS Collaboration, https://atlas.web.cern.ch/Atlas/GROUPS/PHYSICS/CombinedSummaryPlots/SUSY/.

[14] CMS Collaboration, https://twiki.cern.ch/twiki/bin/view/CMSPublic/PhysicsResultsSUS.

[15] N. Arkani-Hamed et al., The Littlest Higgs, JHEP 0207(2002) 034.

[16] D. B. Kaplan, H. Georgi and S. Dimopoulos, Composite Higgs Scalars, Phys. Lett. B 136 (1984) 187.

[17] J. A. Aguilar-Saavedra, Identifying top partners at LHC, JHEP 0911 (2009) 030.

[18] J. L. Rosner, Prominent decay modes of a leptophobic Z0, Phys. Lett. B 387 (1996) 113.

[19] C.T.Hill, Topcolor: Top quark condensation in a gauge extension of the standard model, Phys. Lett. B 266 (1991) 419.

[20] P. H. Frampton and S. L. Glashow, Chiral color: An alternative to the standard model, Phys. Lett. B 190 (1987) 157.

[21] L. Randall and R. Sundrum, Large Mass Hierarchy from a Small Extra Dimension Phys. Rev. Lett. 83 (1999) 3370.

[22] ATLAS Collaboration, Boosted hadronic top identification at ATLAS for early $13 \mathrm{TeV}$ data, ATL-PHYS-PUB-2015-053.

[23] CMS Collaboration, Top Tagging with New Approaches, CMS-PAS-JME-15-002.

[24] CMS Collaboration, Performance of b tagging at sqrt $(s)=8 \mathrm{TeV}$ in multijet, ttbar and boosted topology events CMS-PAS-B2G-13-001.

[25] ATLAS Collaboration Identification of Boosted, Hadronically-Decaying $W$ and $Z$ Bosons in $\sqrt{s}=13 T e V$ Monte Carlo Simulations for ATLAS, ATL-PHYS-PUB-2015-033.

[26] ATLAS Collaboration Identification of Boosted $H \rightarrow b \bar{b}$ Boson Identification with the ATLAS Detector at $\sqrt{s}=13 T e V$, ATL-PHYS-PUB-2015-035.

[27] CMS Collaboration, Identification techniques for highly boosted W bosons that decay into hadrons, JHEP 12 (2014) 017.

[28] ATLAS Collaboration, Search for production of vector-like top quark pairs and of four top quarks in the lepton-plus-jets final state in pp collisions at $\sqrt{s}=13 \mathrm{TeV}$ with the ATLAS detector, ATLAS-CONF-2016-013.

[29] CMS Collabration, Search for pair production of vector-like T quarks in the lepton plus jets final state, CMS-PAS-B2G-16-002.

[30] CMS Collabration, Search for single production of a vector-like T quark decaying to a Higgs boson and a leptonically decaying top quark, CMS-PAS-B2G-15-008.

[31] CMS Collabration, Search for top quark partners with charge $5 / 3$ at $\sqrt{s}=13 T e V$, CMS-PAS-B2G-15-006.

[32] ATLAS Collaboration, Search for heavy particles decaying to pairs of highly-boosted top quarks using lepton-plus-jets events in proton-proton collisions at $\sqrt{s}=13 T e V$ with the ATLAS detector, ATLAS-CONF-2016-014 
[33] CMS Collaboration, Search for $t \bar{t}$ resonances in boosted semileptonic final states in pp collisions at $\sqrt{s}=13 T e V$, CMS-PAS-B2G-15-002.

[34] CMS Collabration, Search for $W$ ' boson resonances decaying into a top quark and a bottom quark in the leptonic final state at $\sqrt{\mathrm{s}}=13 \mathrm{TeV}$, CMS-PAS-B2G-15-004.

[35] CMS Collabration, Search for W' decaying to tau lepton and neutrino in proton-proton collisions at $\sqrt{s}=13 \mathrm{TeV}$, CMS-PAS-B2G-16-006.

[36] M. Fairbairn et al., Stable massive particles at colliders, Phys.Rept. 438 (2007) 1-63.

[37] ATLAS Collaboration, Search for metastable heavy charged particles with large ionization energy loss in pp collisions at $\sqrt{s}=13 \mathrm{TeV}$ using the ATLAS experiment, arXiv:1604.04520.

[38] CMS Collaboration, Searches for Long-lived Charged Particles in Proton-Proton Collisions at $\sqrt{s}=13 \mathrm{TeV}$, CMS-PAS-EXO-15-010.

[39] ATLAS Collaboration, Search for charginos nearly mass degenerate with the lightest neutralino based on a disappearing-track signature in pp collisions at $\sqrt{s}=8 \mathrm{TeV}$ with the ATLAS detector, PRD 88, 112006 (2013).

[40] CMS Collaboration, Search for disappearing tracks in proton-proton collisions at $\sqrt{s}=8 \mathrm{TeV}$, JHEP 01(2015) 096.

[41] ATLAS Collaboration, Search for pair-produced long-lived neutral particles decaying to jets in the ATLAS hadronic calorimeter in pp collisions at $\sqrt{s}=8 \mathrm{TeV}$, PLB 743 (2015) 15-34.

[42] ATLAS Collaboration, Search for long-lived, weakly interacting particles that decay to displaced hadronic jets in proton-proton collisions at $\sqrt{s}=8 \mathrm{TeV}$ with the ATLAS detector, PRD 92, 012010 (2015).

[43] CMS Collaboration, Search for long-lived particles that decay into final states containing two electrons or two muons in proton-proton collisions at $\sqrt{s}=8 \mathrm{TeV}$, PRD 91 (2015) 052012.

[44] CMS Collaboration, Search for long-lived neutral particles decaying to quark-antiquark pairs in proton-proton collisions at $\sqrt{s}=8 \mathrm{TeV}$, PRD 91 (2015) 012007.

[45] ATLAS Collaboration, Search for long-lived neutral particles decaying into lepton jets in proton-proton collisions at $\sqrt{s}=8 \mathrm{TeV}$ with the ATLAS detector, JHEP 11(2014) 088.

[46] CMS Collaboration, Search for pair production of third-generation scalar leptoquarks and top squarks in proton-proton collisions at $\sqrt{s}=8 \mathrm{TeV}$, PLB 739 (2014) 229. 